\title{
PATHOGENIC AND MORPHOLOGICAL VARIABILITY OF Plasmopara halstedii, THE CAUSAL AGENT OF DOWNY MILDEW IN SUNFLOWER
}

\author{
Kulkarni, S. , Hegde, Y.R. and Kota, R.V.
}

Department of Plant Pathology, University of Agricultural Sciences, Dharwad-580 005, Karnataka, India

Received: August 10, 2006

Accepted: March 12, 2009

\section{SUMMARY}

Isolates of $P$. halstedii collected from different parts of Karnataka, Maharashtra and Andhra Pradesh belong to a single race, i.e., race 100. Based on virulence pattern, they can be grouped into five different groups, A, B, C, D and $\mathrm{E}$. Bangalore isolate was most virulent and Bidar isolate was least virulent. Morphological variations existed among the isolates with regard to the size of sporangiophores and sporangia. Sporangiophores were longer Dharwad isolate and sporangia were larger in Mysore and Latur isolates.

Key words: Plasmopara halstedii, downy mildew, pathogenic variability

\section{INTRODUCTION}

Sunflower (Helianthus annuus L.) is an important source of vegetable oils and it keeps gaining popularity because of its high oil percentage and quality, short duration and thermophotinsensitiveness. More than 30 diseases have been identified on sunflower (Gulya et al., 1994). Among these, downy mildew caused by Plasmopara halstedii (Farl.) Berl. and de Toni is the most destructive one (Kolte, 1985). Existence of physiologic races in $P$. halstedii is evident from reports of several workers from different parts of the world (Abdullah, 1983; Patil and Mayee, 1990). However, there is no proper information available about race pattern of $P$. halstedii and hence an attempt was made to generate information about physiologic races of $P$. halstedii.

\section{MATERIAL AND METHODS}

Twelve isolates were collected from different places and designated $\mathrm{Ph} 1$ to $\mathrm{Ph}$ 12 (Table 1). These isolates were maintained and multiplied on the susceptible cul-

* Corresponding author 
tivar "Morden" using the radicle inoculation method. To segregate the race patterns, these isolates were inoculated on 15 international host differentials viz., HA 300, RHA 266A, RHA 325 R, HA 61, RHA 274R, HA 335, HA 337, RHA 340R, DM2, HIR34, HAR, HAR5, DM4, DM5 and DM6. Seeds of host differentials were germinated in rolled paper towels. When radicles were about 3-4 $\mathrm{mm}$ in size, they were atomizer-sprayed with a fresh sporangial suspension prepared in sterile water. Inoculated seeds in the rolled paper towels were incubated at a temperature of $15^{\circ} \mathrm{C}$ for $48 \mathrm{~h}$ and symptom development was observed.

Table 1: Designations of the twelve Plasmopara halstedii isolates

\begin{tabular}{lll}
\hline No. & Locality of collection of isolates & Designation of isolate \\
\hline 1 & Gadag & $P h 1$ \\
2 & Bangalore & $P h 2$ \\
3 & Dharwad & $P h 3$ \\
4 & Mysore & $P h 4$ \\
5 & Narendra & $P h 5$ \\
6 & Latur & $P h 6$ \\
7 & Hyderabad & $P h 7$ \\
8 & Bailhongal & $P h 8$ \\
9 & Davanagere & $P h 9$ \\
10 & Haveri & $P h 10$ \\
11 & Bidar & $P h 11$ \\
12 & Parabhani & $P h 12$ \\
\hline
\end{tabular}

\section{Virulence index of $P$. halstedii isolates}

Virulence index was calculated by inoculating the susceptible cv. Morden. Observations were made on percent disease incidence and latent period required for expression of symptoms. Further, virulence index for each isolate was calculated using the following formula.

$$
\text { virulence index }=\text { percent incidence } \times \frac{1}{\text { latent period }}
$$

\section{RESULTS AND DISCUSSION}

It is evident from Table 2 that all of the collected isolates successfully produced the disease on HA300, which has no resistance genes. All isolates produced disease on true leaves of HA300. All other differentials were free from infection on true leaves although sporulation was observed on HA335 and HAR5 at the cotyledon stage. The obtained reaction was similar to race 100 or European race 1 (Table 3 ). It can be noted that the reaction was similar in all isolates collected, which is an indication that all the isolates belong to a single race, i.e., race 100 . This may be due to a limited spread of the pathogen, its homothallic nature and the narrow 
genetic base of the sunflower genotypes grown in India. Similarly, Patil and Mayee (1990) reported that the sunflower downy mildew isolate from Latur was found to be the European race 1 (race 100).

Table 2: Reaction of different Plasmopara halstedii isolates on host differentials of sunflower

\begin{tabular}{|c|c|c|c|c|c|c|c|c|c|c|c|c|c|}
\hline \multirow{2}{*}{$\begin{array}{l}\text { Sl. } \\
\text { No. }\end{array}$} & \multirow{2}{*}{ Differential } & \multicolumn{12}{|c|}{ Isolate } \\
\hline & & $\overline{P h 1}$ & $P h 2$ & $P h 3$ & $P h 4$ & $P h 5$ & $P h 6$ & $P h 7$ & $P h 8$ & $P h 9$ & $P h 10$ & $P h 11$ & $P h 12$ \\
\hline 1 & HA 300 & $S$ & $S$ & $S$ & $S$ & $S$ & $S$ & $S$ & $S$ & $S$ & $S$ & $S$ & $S$ \\
\hline 2 & RHA 266 R & $\mathrm{R}$ & R & $\mathrm{R}$ & R & $\mathrm{R}$ & $\mathrm{R}$ & $\mathrm{R}$ & $\mathrm{R}$ & $R$ & $\mathrm{R}$ & $\mathrm{R}$ & R \\
\hline 3 & RHA $325 \mathrm{R}$ & $\mathrm{R}$ & $\mathrm{R}$ & $R$ & $R$ & $R$ & $\mathrm{R}$ & $\mathrm{R}$ & $R$ & $R$ & $\mathrm{R}$ & $\mathrm{R}$ & $\mathrm{R}$ \\
\hline 4 & HA 61 & $\mathrm{R}$ & $\mathrm{R}$ & $R$ & $R$ & $R$ & $\mathrm{R}$ & $\mathrm{R}$ & $R$ & $R$ & $R$ & $\mathrm{R}$ & $R$ \\
\hline 5 & RHA 274 R & $\mathrm{R}$ & $\mathrm{R}$ & $\mathrm{R}$ & $R$ & $R$ & $\mathrm{R}$ & $\mathrm{R}$ & $R$ & $R$ & $R$ & $\mathrm{R}$ & $\mathrm{R}$ \\
\hline 6 & HA 335 & $\mathrm{R}$ & $R$ & $\mathrm{R}$ & $R$ & $\mathrm{R}$ & $\mathrm{R}$ & $\mathrm{R}$ & $\mathrm{R}$ & $R$ & $\mathrm{R}$ & $\mathrm{R}$ & $R$ \\
\hline 7 & HA 337 & $\mathrm{R}$ & $\mathrm{R}$ & $R$ & $R$ & $R$ & $\mathrm{R}$ & $R$ & $R$ & $R$ & $R$ & $\mathrm{R}$ & $\mathrm{R}$ \\
\hline 8 & $\mathrm{RHA} 340 \mathrm{R}$ & $\mathrm{R}$ & $\mathrm{R}$ & $\mathrm{R}$ & $R$ & $\mathrm{R}$ & $\mathrm{R}$ & R & R & $R$ & $\mathrm{R}$ & $\mathrm{R}$ & $R$ \\
\hline 9 & DM 2 & $\mathrm{R}$ & $\mathrm{R}$ & $R$ & $R$ & $R$ & $\mathrm{R}$ & $\mathrm{R}$ & $R$ & $R$ & $\mathrm{R}$ & $\mathrm{R}$ & $R$ \\
\hline 10 & HI R 34 & $\mathrm{R}$ & $\mathrm{R}$ & $\mathrm{R}$ & R & R & $\mathrm{R}$ & $\mathrm{R}$ & R & R & $\mathrm{R}$ & $\mathrm{R}$ & $\mathrm{R}$ \\
\hline 11 & HA R4 & $\mathrm{R}$ & $\mathrm{R}$ & $R$ & $R$ & $R$ & $\mathrm{R}$ & $\mathrm{R}$ & $R$ & $R$ & $\mathrm{R}$ & $\mathrm{R}$ & $\mathrm{R}$ \\
\hline 12 & HA R5 & $\mathrm{R}$ & $\mathrm{R}$ & $\mathrm{R}$ & $\mathrm{R}$ & $\mathrm{R}$ & $\mathrm{R}$ & $\mathrm{R}$ & R & R & R & $\mathrm{R}$ & $\mathrm{R}$ \\
\hline 13 & DM 4 & $\mathrm{R}$ & $\mathrm{R}$ & $\mathrm{R}$ & $\mathrm{R}$ & $\mathrm{R}$ & $\mathrm{R}$ & $\mathrm{R}$ & $\mathrm{R}$ & $R$ & $\mathrm{R}$ & $\mathrm{R}$ & $\mathrm{R}$ \\
\hline 14 & DM 5 & $\mathrm{R}$ & $R$ & $\mathrm{R}$ & $\mathrm{R}$ & $\mathrm{R}$ & $R$ & $\mathrm{R}$ & R & R & $\mathrm{R}$ & $\mathrm{R}$ & $\mathrm{R}$ \\
\hline 15 & DM 6 & $\mathrm{R}$ & $\mathrm{R}$ & $\mathrm{R}$ & $\mathrm{R}$ & $\mathrm{R}$ & $\mathrm{R}$ & $\mathrm{R}$ & $\mathrm{R}$ & $\mathrm{R}$ & $\mathrm{R}$ & $\mathrm{R}$ & $\mathrm{R}$ \\
\hline
\end{tabular}

Table 3: List of international host differentials of sunflower selected for race identification studies, genes present and their reactions to the identified races of Plasmopara halstedii.

\begin{tabular}{|c|c|c|c|c|c|c|c|c|c|}
\hline \multirow{2}{*}{ No. } & \multirow{2}{*}{ Differential } & \multirow{2}{*}{$\begin{array}{l}\text { Resistant gene/s } \\
\text { present }\end{array}$} & \multicolumn{7}{|c|}{ Race reaction } \\
\hline & & & 1 & 2 & 3 & 4 & 5 & 6 & 7 \\
\hline 1 & HA 300 & - & $S$ & $S$ & $S$ & $S$ & $S$ & $S$ & $S$ \\
\hline 2 & RHA 266 R & $\mathrm{Pl} 1$ & $R$ & S & S & S & S & S & S \\
\hline 3 & RHA 325 R & $P I 2, P l b$ & $R$ & $R$ & S & S & S & S & $\mathrm{R}$ \\
\hline 4 & HA 61 & $P / 2,3+?$ & $R$ & $\mathrm{R}$ & S & S & S & $\mathrm{R}$ & $\mathrm{R}$ \\
\hline 5 & RHA 274 R & $P I 2,9, P l b$ & $R$ & $\mathrm{R}$ & S & s & S & $\mathrm{R}$ & $\mathrm{R}$ \\
\hline 6 & HA 335 & PI6 +? & $R$ & $\mathrm{R}$ & $R$ & $R$ & $R$ & $\mathrm{R}$ & $\mathrm{R}$ \\
\hline 7 & HА 337 & $P / 7+?$ & $R$ & $\mathrm{R}$ & $R$ & $R$ & $R$ & $\mathrm{R}$ & $\mathrm{R}$ \\
\hline 8 & RHA 340 R & $P / 8+?$ & $R$ & $R$ & $R$ & $R$ & $\mathrm{R}$ & $\mathrm{R}$ & $\mathrm{R}$ \\
\hline 9 & DM 2 & $P / 2,5$ & $R$ & $R$ & $R$ & S & S & S & S \\
\hline 10 & HI R 34 & $P / 4+?$ & $\mathrm{R}$ & $R$ & $S$ & S & $S$ & $\mathrm{R}$ & $\mathrm{R}$ \\
\hline 11 & HA R4 & Plf $+?$ & $R$ & $R$ & $R$ & $\mathrm{R}$ & $\mathrm{R}$ & $\mathrm{R}$ & $\mathrm{R}$ \\
\hline 12 & HA R5 & $P l g+?$ & $R$ & $R$ & $R$ & $R$ & $\mathrm{R}$ & $\mathrm{R}$ & $\mathrm{R}$ \\
\hline 13 & DM 4 & $P l c+?$ & $\mathrm{R}$ & $\mathrm{R}$ & $\mathrm{R}$ & $\mathrm{R}$ & S & $\mathrm{R}$ & $S$ \\
\hline 14 & DM 5 & $P l d+?$ & $\mathrm{R}$ & $\mathrm{R}$ & $\mathrm{R}$ & $\mathrm{R}$ & $S$ & $\mathrm{R}$ & $\mathrm{R}$ \\
\hline 15 & DM 6 & $\mathrm{Ple}+?$ & $\mathrm{R}$ & $\mathrm{R}$ & $\mathrm{R}$ & S & S & $\mathrm{R}$ & $\mathrm{R}$ \\
\hline
\end{tabular}


Table 4: Morphological variations among Plasmopara halstedii isolates

\begin{tabular}{|c|c|c|c|c|}
\hline No. & Isolate & $\begin{array}{l}\text { Sporangiophore } \\
\text { length }(\mu \mathrm{m})\end{array}$ & $\begin{array}{l}\text { Sporangiophore } \\
\text { width }(\mu \mathrm{m})\end{array}$ & Sporangial size $(\mu \mathrm{m})$ \\
\hline 1 & Gadag (Ph 1) & $487-770(602)$ & $5.00-12.50(9.05)$ & $\begin{array}{c}12.50-27.50 \times 12.50-17.50 \\
(21.16 \times 18.00)\end{array}$ \\
\hline 2 & Bangalore (Ph 2) & $460-690(595)$ & $6.50-13.50(8.95)$ & $\begin{array}{c}14.30-26.30 \times 14.15-28.61 \\
(20.65 \times 17.97)\end{array}$ \\
\hline 3 & Dharwad (Ph 3) & $575-675(620)$ & $7.50-10.00(8.63)$ & $\begin{array}{c}17.50-25.00 \times 15.00-20.65 \\
(21.00 \times 18.16)\end{array}$ \\
\hline 4 & Mysore (Ph 4) & $525-650(581)$ & $10.00-15.00(12.16)$ & $\begin{array}{c}17.50-27.50 \times 17.50-22.50 \\
(25.16 \times 19.33)\end{array}$ \\
\hline 5 & Narendra $(P h 5)$ & $325-650(485)$ & $7.50-10.0(8.12)$ & $\begin{array}{c}17.50-27.50 \times 15.00-20.00 \\
(20.83 \times 17.66)\end{array}$ \\
\hline 6 & Latur $(P h 6)$ & $557-612$ (595) & $10.00-12.50(11.05)$ & $\begin{array}{c}22.50-40.00 \times 15.00-22.50 \\
(25.17 \times 17.67)\end{array}$ \\
\hline 7 & Hyderabad $(P h 7)$ & $460-695(580)$ & $6.50-13.50(8.92)$ & $\begin{array}{c}18.15-28.45 \times 15.00-20.00 \\
(22.30 \times 17.50)\end{array}$ \\
\hline 8 & Bailhongal (Ph 8) & $325-690(565)$ & $6.00-13.55(8.15)$ & $\begin{array}{c}18.35-24.50 \times 16.10 \times 21.60 \\
(21.85 \times 19.35)\end{array}$ \\
\hline 9 & Davanagere $(P h 9)$ & $470-630(570)$ & $5.00-12.5$ (8.95) & $\begin{array}{c}14.65-27.30 \times 15.00-21.00 \\
(20.10 \times 18.16)\end{array}$ \\
\hline 10 & Haveri $(P h 10)$ & $430-685(565)$ & $7.50-10.00(8.30)$ & $\begin{array}{c}17.85-26.50 \times 15.89-21.50 \\
(20.53 \times 18.65)\end{array}$ \\
\hline 11 & Bidar (Ph 11) & $470-630(585)$ & $5.00-13.00(9.00)$ & $\begin{array}{c}15.00-25.65 \times 14.65 \times 21.30 \\
(21.63 \times 18.01)\end{array}$ \\
\hline 12 & Parbhani (Ph 12) & $470-630(565)$ & $10.00-15.00(11.05)$ & $\begin{array}{c}14.30-27.50 \times 15.00-22.50 \\
(20.65 \times 17.67)\end{array}$ \\
\hline
\end{tabular}

Numbers in parentheses are mean values.

Table 5: Virulence index (percent incidence $\times$ latent $_{\text {period }}{ }^{-1}$ ) of different Plasmopara halstedii isolates

\begin{tabular}{lll}
\hline No. & Isolate & Virulence index * \\
\hline 1 & Gadag $(P h ~ 1)$ & 8.60 \\
2 & Bangalore $(P h$ 2) & 12.11 \\
3 & Dharwad $(P h$ 3) & 7.79 \\
4 & Mysore $(P h$ 4) & 10.17 \\
5 & Narendra $(P h$ 5) & 7.72 \\
6 & Latur $(P h$ 6) & 9.81 \\
7 & Hyderabad $(P h ~ 7)$ & 10.20 \\
8 & Bailhongal $(P h$ 8) & 7.85 \\
9 & Davanagere $(P h ~ 9)$ & 8.69 \\
10 & Haveri $(P h ~ 10)$ & 7.92 \\
11 & Bidar $(P h ~ 11)$ & 6.26 \\
12 & Parbhani $(P h ~ 12)$ & 8.90 \\
\hline & SEm \pm & 0.24 \\
& CD (1\%) & 0.70 \\
\hline
\end{tabular}

\footnotetext{
${ }^{\star}$ Average of three replications
} 


\section{Morphological variability}

Mean sporangiophore length was highest in Dharwad (Ph 3) isolate (620 $\mu \mathrm{m})$. The sporangiophore length ranged between 325 and $770 \mu \mathrm{m}$. Sporangiophore width was largest in Mysore $(\mathrm{Ph} 4)$ isolate. Sporangial size varied from $12.50 \times$ $12.50 \mu \mathrm{m}$ to $40.0 \times 22.50 \mu \mathrm{m}$. The mean sporangial size was highest in Mysore $(\mathrm{Ph}$ 4 ) and Latur ( $P h$ 6) isolates (Table 4).

\section{Virulence index}

Results shown in Table 5 indicated that Bangalore ( $P h$ 2) isolate was most virulent among the isolates tested with a virulence index of 12.11 , followed by Hyderabad isolate $(P h 7)$ with a virulence index of 10.20. Bidar isolate was least virulent, recording the lowest virulence index of 6.26. Based on the virulence pattern of the isolate, the isolates were grouped into five groups as shown below.

\begin{tabular}{ll}
\hline Virulence group & $P$. halstedii isolate \\
\hline Group A & $P h 2$ \\
Group B & $P h 4, P h 6, P h 7$ \\
Group C & $P h 1, P h 9, P h 12$ \\
Group D & $P h 3, P h 5, P h 8, P h 10$ \\
Group E & $P h 11$ \\
\hline
\end{tabular}

\section{ACKNOWLEDGEMENT}

Authors are thankful to the Indian Council of Agricultural Research, New Delhi, for providing financial assistance for this investigation.

\section{REFERENCES}

Abdullah, M.T., 1984. Sunflower hybrids resistant to Red River races of downy mildew. Proceedings of XI International Sunflower Conference, Mar De Plata, Argentina 89.

Gulya, T., Berlin, N. and Lamey, A., 1994. Sunflower disease. In: Sunflower Production Ext. Bulletin. North Dakota. Agric. Experiment Station and North Dakota State Univ., pp. 4462.

Kolte, S.J., 1985. Diseases of Annual Edible Oilseed Crops. Vol. III Sunflower, Safflower and Niger Seed Diseases, CRC Press Inc., Florida, USA, p. 154.

Patil, M.A. and Mayee, C.D., 1990, Race identification of Indian P. halstedii, cause of downy mildew of sunflower. Indian Phytopathology 43: 517-519. 


\title{
ESTBRE LA PATOGENOCIDAD Y VARIABILIDAD MORFOLÓGICA EN Plasmopara halstedii, AGENTE CAUSAL DEL DOWNY MILDEW DEL GIRASOL
}

\author{
RESUMEN
}

Las aislaciones de $P$. halstedii provenientes de diferentes partes de Karnataka, Maharashtra y Andhra Pradesh pertenecen a una sola raza (raza 100). Sobre la base de patrones de virulencia, éstas pueden ser agrupadas en cinco diferentes grupos denominados A, B, C, D y E. El aislamiento de Bangalore fue el más virulento mientras que el de Bidar fue el de menor virulencia. Se observaron variaciones morfológicas entre aislamientos en relación al tamaño de los esporangióforos y los esporangios. El aislamiento de Dharward presentó los esporangióforos más largos mientras que los aislamientos de Mysore y Latur presentaron los esporangios más grandes.

\section{ÉTUDE DE LA VARIABILITÉ PATHOGÈNE ET MORPHOLOGIQUE DE LA SORTE Plasmopara halstedii, CAUSE DE LA ROUILLE CHEZ LE TOURNESOL}

\author{
RÉSUMÉ
}

Des isolats de $P$. halstedii collectés dans différentes parcelles de Karnataka, Maharashtra et Andhra Pradesh appartiennent à une seule race, à savoir la race 100 . La quantification de l'agressivité a conduit à l'identification de 5 groupes (A à E): l'isolat de Bangalore a été le plus agressif, et celui de Bidar le moins agressif. Des variations de longueur des sporangiophores et des sporanges ont été notées: la longueur des sporangiophores de l'isolat de Dharwad était la plus importante, tandis que les tailles des sporanges des isolats de Mysore et Latur étaient les plus importantes. 